Pteridines

Vol. 1, 1989, pp. $37-43$

\title{
Insights into Enzymic Catalysis from Studies on Dihydrofolate Reductases
}

\author{
By Stephen J. Benkovic*, Joseph A. Adams \\ Department of Chemistry, The Pennsylvania State University, University Park, PA 16802, U.S.A. \\ Carol A. Fierke \\ Duke University Medical Center, Biochemistry Department, Box 3711, Durham, N.C. 27710, U.S.A.
}

Adel M. Naylor

California Institute of Technology, Department of Chemistry, California Institute of Technology, Pasadena, CA 91125 , U.S.A.

(Received September 1988)

\begin{abstract}
Summary
The role of DHFR in the maintenance of cellular DNA has sparked wide interest in the structure and dynamics of this enzyme. Kinetic studies of specific amino acid replacements on the enzyme isolated from $E$. coli has proved useful in the detailing of hydrophobic and ionic interactions both proximal and distal to the site of chemical transformation (e. g. Phe-31, Leu-54 and Arg-44). Despite the low sequence homology shared by the E. coli and $L$. casei enzymes, the free energy profiles are surprisingly comparable. This probably is the result of the high degree of structural similarity of the active site surfaces, but the deleterious effects of subtle replacements (e. g. Leu-54-Ile) at strictly conserved amino acids underscore the latters unique role in attaining the required catalytic efficiency for the enzyme.
\end{abstract}

\section{Introduction}

Dihydrofolate reductase $(5,6,7,8$-tetrahydrofolate: $\mathrm{NADP}^{+}$oxidoreductase, EC 1.5.1.3) catalyses the NADPH-dependent reduction of 7,8-dihydrofolate $\left(\mathrm{H}_{2} \mathrm{~F}\right)$ to $5,6,7,8$-tetrahydrofolate $\left(\mathrm{H}_{4} \mathrm{~F}\right)$. The enzyme is essential for both eucaryotes and procaryotes to avoid depletion of $\mathrm{H}_{4} \mathrm{~F}$ owing to its oxidation by thymidylate synthetase and thus directly influences pyrimidine biosynthesis. It has long been the target enzyme for chemotherapeutic agents and its inhibition by methotrexate (MTX) is mainly responsible for the mechanism of action of this drug (1). Because of its biological and pharmacological importance, dihydrofolate reductase (DHFR) has been the subject of

\footnotetext{
* Author to whom correspondence should be addressed.
} Abbreviations: DHFR, dihydrofolate reductase; $\mathrm{H}_{2} \mathrm{~F}, 7,8$-dihydrofolate; $\mathrm{H}_{4} \mathrm{~F}$ 5,6,7,8-tetrahydrofolate; MTX, methotrexate. BIOGRAF* is an interactive molecular simulation 3-dimensional graphics program from BIODESIGN, Inc., Pasadena, CA.

Pteridines / Vol. 1 / 1989 / No.

Copyright (C) 1989 Walter de Gruyter · Berlin · New York extensive structural and mechanistic investigations. In particular the X-ray crystallography has provided the structures of the MTX and MTX - NADPH enzyme complexes for the $E$. coli and $L$. case $i$ species respectively $(2-5)$. Stereochemistry has traced the course of the reduction and established that $\mathrm{H}_{2} \mathrm{~F}$ must be bound in a conformation in which the pteridine ring is rotated $180^{\circ}$ relative to the $\mathrm{C} 6-\mathrm{C} 9$ bond of the side chain in MTX (6). With the development of the protocols for site specific mutagenesis, it has become possible to pursue several novel lines of inquiry: i) to evaluate the role of hydrophobic residues in catalysis and in substrate and drug binding; ii) to ascertain the importance of proximal and distal residues on the acidity and function of the unique active site carboxylate; and iii) to determine the influence of multiple amino acid changes on the thermodynamic and kinetic characteristics of the active site by comparing the $E$. coli and $L$. casei enzymes. 
<smiles>[X]CC1CNc2nc(N)[nH]c(=O)c2N1</smiles><smiles>CNc1ccc(C(=O)NC(CCC(=O)O)C(=O)O)cc1</smiles>

\section{Structural and Kinetic Features of Native DHFR}

The active site structure of the E. coli DHFR - methotrexate - NADPH complex along with several conserved amino acid residues that have been altered by mutagenesis is depicted in figure 1. The NADPH has been positioned based on analogy to its observed locus in the $L$. casei complex. The active site is a cavity some $15 \AA$ deep lined by hydrophobic side chains with the only acidic function present being the carboxyl of Asp-27. NADPH binds in an extended conformation with the nicotinamide moiety inserted through the entrance of the cavity and the pyrophosphoryl linkage making strong ionic and hydrogen bonding contacts

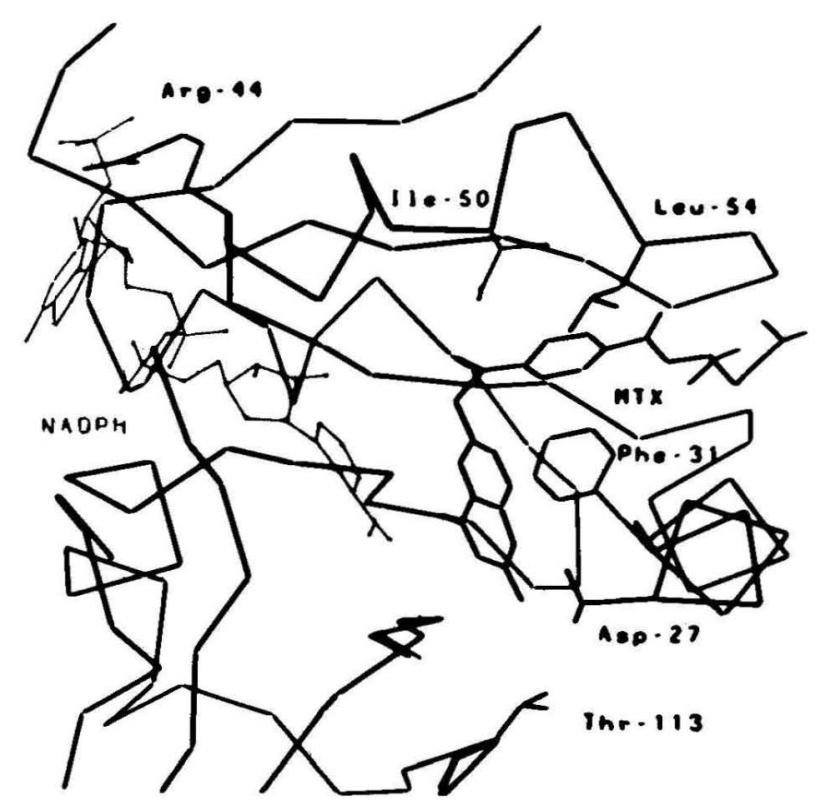

Figure 1. Carbon backbone structure of E. coli DHFR - MTX - NADPH and the side chains for key amino acids depicted using BIOGRAF*.
(Arg-44, His-45). MTX, and by inference $\mathrm{H}_{2} \mathrm{~F}$, lies towards the back of the site where the pteridine and benzoyl rings are surrounded by hydrophobic residues (e. g. Ile-5, Phe-31, Ile-50, and Leu-54). The Asp-27 interacts with the $\mathrm{N} 2$ and $\mathrm{N} 3$ nitrogens of the pyrimidine ring and does not make a direct hydrogen bonded contact with N5, despite the fact that protonation of $\mathrm{N} 5$ is required to complete the reduction of the N5-C6 imine upon hydride transfer to C6.

The kinetic sequence for $E$. coli DHFR that predicts accurately the observed steady-state kinetic parameters and full-time course kinetics under a variety of substrate concentrations and as a function of $\mathrm{pH}$ was estblished by measuring the various ligand association and dissociation rate constants and pre-steady-state reaction transients employing stopped-flow fluorescence and absorbance spectroscopy (Fig. 2) (7). The enzyme has an intrinsic fluorescence that is quenched by the binding of ligands, particularly MTX. Independent measurements of the equilibrium binding for the various ligand combinations to form the binary and ternary reactant and product complexes gave dissociation constants in satisfactory agreement with those calculated from kinetic measurements of individual rate constants implying that the kinetic scheme is self consistent. Several features of the kinetic sequence germane to our considerations are: i) steadystate turnover is limited by $\mathrm{H}_{4} \mathrm{~F}$ release; ii) the nonequilibrium binding of $\mathrm{H}_{2} \mathrm{~F}$ and NADPH becomes ordered owing to the fact that the rate constant for $\mathrm{H}_{4} \mathrm{~F}$ dissociation is greatest from the mixed ternary E - NADPH $\cdot \mathrm{H}_{4} \mathrm{~F}$ complex rather than either of the two possible product complexes; and iii) the overall reaction strongly favors $\mathrm{H}_{4} \mathrm{~F}$ formation $\left(K_{\mathrm{ov}} \sim 10^{11}\right)$ that is partially reflected in the internal equilibrium, $\mathrm{E} \cdot \mathrm{NADPH} \cdot \mathrm{H}_{2} \mathrm{~F} \rightleftharpoons \mathrm{E} \cdot \mathrm{NADP}^{+} \cdot \mathrm{H}_{4} \mathrm{~F}\left(K_{\mathrm{int}} \sim 10^{3}\right)$. 


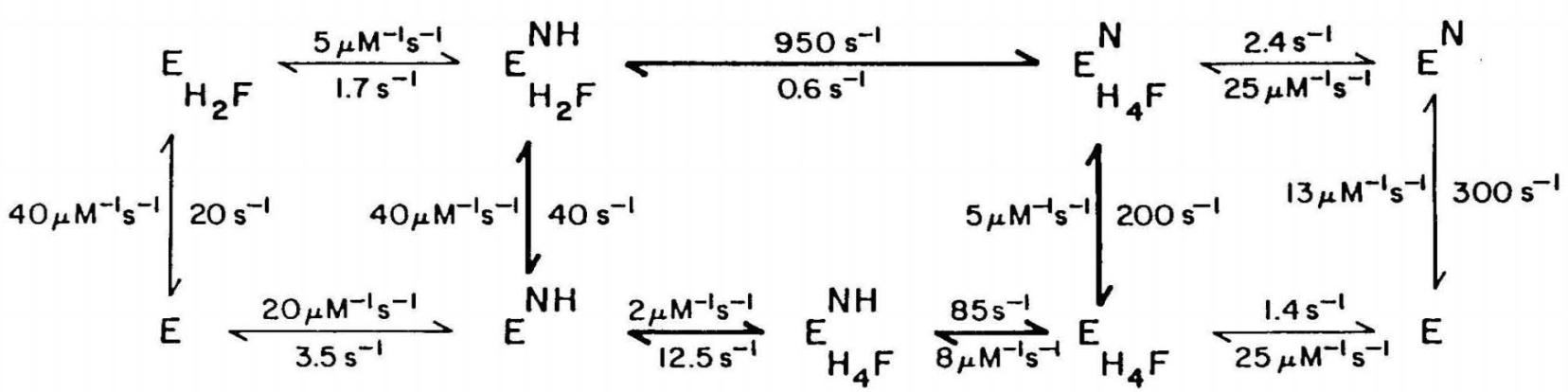

Figure 2. Kinetic scheme for DHFR at $25^{\circ} \mathrm{C}, \mathrm{MTEN}$ buffer, N, NADP; NH, NADPH; $\mathrm{H}_{2} \mathrm{~F}$, dihydrofolate; $\mathrm{H}_{4} \mathrm{~F}$, tetrahydrofolate.

\section{The Active Site Asp-27}

Reasoning from chemical precedent, the mechanism of imine reduction is expected to favor preprotonation at N5 followed by hydride transfer. However the absence of a proximal acid function calls into question this presumption and raises issues concerning the identity of the acidic residue and the rates of proton transfer steps to $\mathrm{H}_{2} \mathrm{~F}$ and medium.

Examination of the $\mathrm{pH}$ dependence of the steadystate turnover showed that the rate limiting step is $\mathrm{H}_{4} \mathrm{~F}$ release at $\mathrm{pH}<7.5$ which changes to the chemical step of hydride transfer at $\mathrm{pH}>8.5$. The $\mathrm{pH}$ $V_{\mathrm{M}}$ transition is controlled by the ionization of an acidic residue with an apparent $\mathrm{p} K_{\mathrm{a}} \sim 8.4$ (Fig. 3). The rate constant for the isolated hydride transfer step is dependent on an acidic active site group with a $\mathrm{p} K_{\mathrm{a}} \sim 6.5$, a value that is the same for all forms of the enzyme: free, binary and ternary complexes. Although binding of $\mathrm{H}_{2} \mathrm{~F} / \mathrm{H}_{4} \mathrm{~F}$ is $\mathrm{pH}$ independent, the

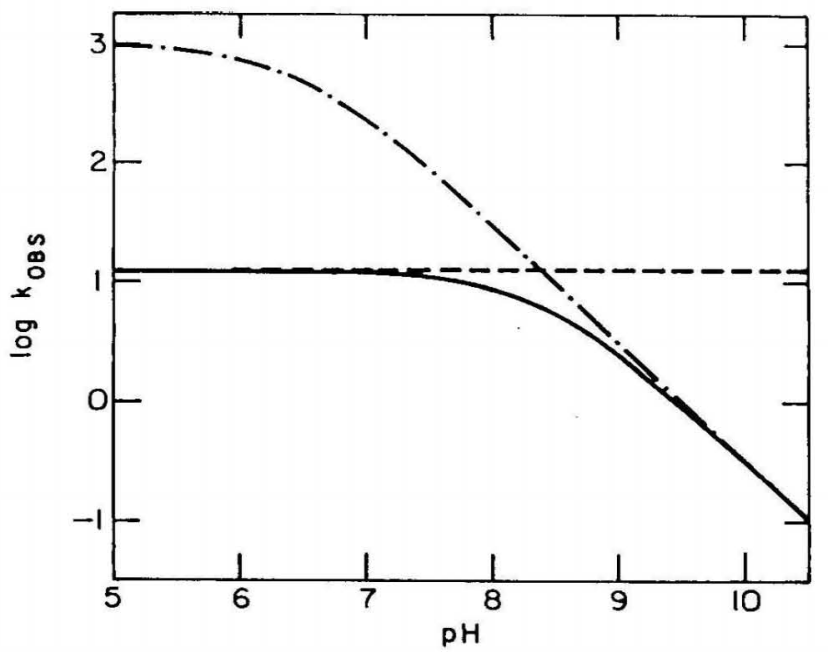

Figure 3. Observed rate constants for hydride transfer $(-\cdot-)$, $\mathrm{H}_{4} \mathrm{~F}$ dissociation (-- ) and $V_{\mathrm{M}}(-)$ as a function of $\mathrm{pH}$. rate of dissociation of $\mathrm{H}_{4} \mathrm{~F}$ is significantly slower than the rate of hydride transfer up to $\mathrm{pH} 8.4$ increasing the $\mathrm{p} K_{\mathrm{a}}$ observed in $V_{\mathrm{M}}$ by ca. $1.9 \mathrm{p} K_{\mathrm{a}}$ units. The identity of the acidic active site residue was clearly shown to be the remote Asp-27 by its replacement with Asn or Ser resulting in two mutant enzyme forms that require preprotonated $\mathrm{H}_{2} \mathrm{~F}$ for activity (8).

Several lines of evidence from wild-type and mutant enzymes suggest that despite its cloistered location at the back of the active site cavity, the proton on Asp27 readily exchanges with solvent namely: deuterium kinetic isotope effects on $V_{\mathrm{M}} / K_{\mathrm{M}}\left(\mathrm{H}_{2} \mathrm{~F}\right)$ and $V_{\mathrm{M}}$ that at high $\mathrm{pH}$ converge to the same value of ca. 3 (9); and the $V_{\mathrm{M}} / K_{\mathrm{M}}\left(\mathrm{H}_{2} \mathrm{~F}\right) \mathrm{pH}$-rate profile for the Tyr-31 mutant that exhibits a "hollow" in the vicinity of the apparent $\mathrm{p} K_{\mathrm{a}}(10)$. Similarly there is no sign that the proton transfer component of the chemical step becomes rate-limiting since i) the amplitude of the hydride transfer step in native DHFR, which is stoichiometric with enzyme, is not attenuated at a $\mathrm{pH}$ where the ternary complex exists equally in both protonated and nonprotonated forms and ii) the perturbed $\mathrm{p} K_{\mathrm{a}}$ $\left(\Delta \mathrm{p} K_{\mathrm{a}} \sim 0-2\right)$ of Asp-27 in various mutants does not correlate with the rate of hydride transfer. Thus, despite its location the carboxyl of Asp-27 can effectively function to donate its proton to N5 probably through a specific water channel or via the periphery of the pterin ring (N3 $\rightarrow$ O5 $\rightarrow \mathrm{N} 5$ ) (11). There is also evidence from mutants of DHFR that the carboxyl can be repositioned, e.g. by substitution of an Asp for Thr-113 and a neutral replacement at position-27. However, the lower catalytic efficiency $\left[V_{\mathrm{M}} / K_{\mathrm{M}}\left(\mathrm{H}_{2} \mathrm{~F}\right)\right]$ cannot be as yet unequivocally attributed to a decreased rate of proton transfer (12).

Pressing our line of inquiry further, we questioned whether the $\mathrm{p} K_{\mathrm{a}}$ of Asp-27, and hence its interaction with $\mathrm{H}_{2} \mathrm{~F}$ and $\mathrm{H}_{4} \mathrm{~F}$, might be coupled to events in the NADPH binding site in order to rationalize the 
NADPH assistance of $\mathrm{H}_{4} \mathrm{~F}$ dissociation. Mutation of Arg-44 to Leu-44 (13) effectively deletes the ionic interaction of this side chain with the 2'-phosphate and pyrophosphate moiety of the nicotinamide cofactor in the binary complex as well as to neighboring residues in the free enzyme (e.g. Pro-66 and Ser-63) and has a profound unexpected influence on the $\mathrm{p} K_{\mathrm{a}}$ of Asp-27 in the free, binary, and ternary enzyme complexes. In all cases the $\mathrm{p} K_{\mathrm{a}}$ is increased by ca. $2 \mathrm{p} K_{\mathrm{a}}$ units to 8.4 (14). Figure 4 traces the amino acid backbone from Arg-44 in the $\mathrm{N}$ terminal direction to Asp-27 and in the C-terminal direction to Arg-52 and Arg-57 - the latter residues form salt bridges to the two glutamate carboxylate residues of MTX. The distance from Arg-44 to Asp-27 is ca. $25 \AA$, suggesting subtle movement of the peptide chain is responsible for the $\mathrm{p} K_{\mathrm{a}}$ change rather than an environmental effect. If the effect were purely electrostatic, a simple coulombic calculation $\left(\Delta \mathrm{p} K_{\mathrm{a}}=244 / D r\right.$ where $D$ is the effective dielectric constant and $r$ is the charge separation distance in $\AA$ ) would require a dielectric constant of 5 (heptane $\sim 4$ ). This is far less than the range of $50-100$ reported for the influence of $\mathrm{mu}$ tations on the $\mathrm{p} K_{\mathrm{a}}$ of His-64 of subtilisin (15). The Leu-44 mutant is also characterized by an accelerating effect by NADPH on the rate of $\mathrm{H}_{4} \mathrm{~F}$ dissociation $\left(>45 \mathrm{~s}^{-1}\right.$ vs. $\left.12 \mathrm{~s}^{-1}\right)$ and a reduced rate for hydride transfer $\left(50 \mathrm{~s}^{-1}\right.$ and $\left.950 \mathrm{~s}^{-1}\right)$, although the deuterium kinetic isotope effect (NADPD) on the hydride step remains the same (14). Thus we conjecture that the molecular origin of the NADPH effect commences at or near Arg-44 and extends to either or both Asp-27 and Arg-52/57 thereby weakening ionic/hydrogen bonding as well as hydrophobic interactions with $\mathrm{H}_{4} \mathrm{~F}$.

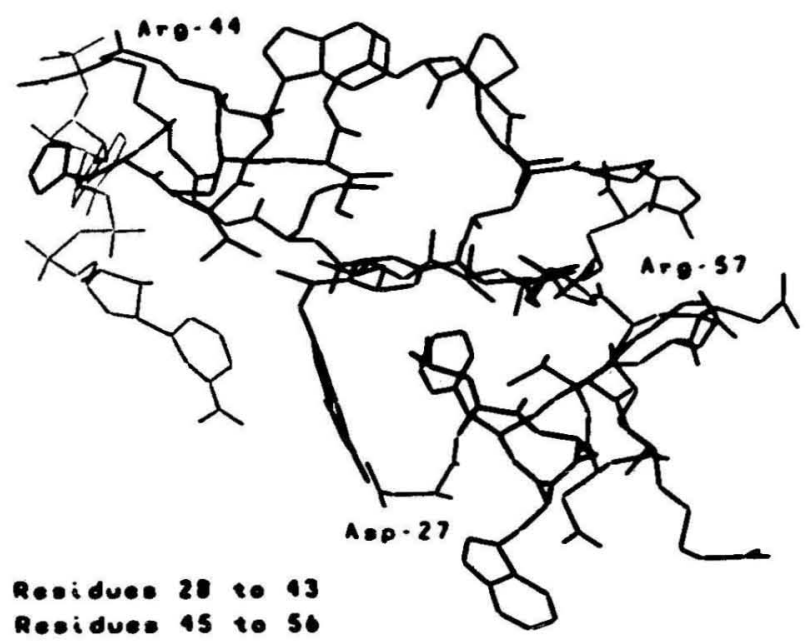

Figure 4. Amino acid structure of $E$. coli from Arg-44 to Arg57 (C-terminal direction) and Asp-27 (N-terminal direction) depicted using BIOGRAF*.
The reduced rate of hydride transfer in this mutant, however, is not a result of an inadequate rate for proton transfer but is probably a consequence of alternate conformations for bound NADPH (14).

\section{Hydrophobic Effects}

In the study of designed mutant proteins whose purpose is to evaluate specific interactions, i. e. hydrogen bonds, ionic pairs or hydrophobic effects, a recurring question is their structural integrity versus the wildtype. Crystallographic studies on DHFR E. coli mutants (Asn-27 and Ser-27) showed that neither the MTX binding geometry nor the detailed three-dimensional topography of the enzyme was altered within $\pm 0.2 \AA(8)$. However a substitution, be it a conservative deletion or replacement, will lead to conformational changes that may be localized or subtly propagated through the protein. Since in estimating the value of a side chain contribution to the free energy of binding the most relevant comparison is its deletion to a glycine, which because of its reduced volume, most certainly will be solvated differently at the active site. Thus in general $\Delta \Delta G_{\text {obs }}$ may not be equated to the binding energy of the moiety in question, although this term may be the predominant contribution (16). With that caveat one can still assess the apparent binding energy and the relative contribution of a given residue to the active site ensemble.

It has been particularly instructive to examine the effect of a mutation (Phe-31 to Val-31) on the binding of the drug MTX. MTX is an example of a slow binding inhibitor where the bimolecular association proceeds in two stages via an initial encounter complex that isomerizes to the observed structure $(17,18)$. On the presumption that the free energies of the wildtype and mutant enzymes are equal, a free energy diagram has been constructed for the binding of MTX to the wild-type DHFR plus two mutants at Phe-31 (19). Several salient results emerged: i) replacement of Phe-31 by Val-31 alters the accessibility of MTX to its binding pocket by reducing the bimolecular combination rate a hundred fold; ii) the value of edge to face aromatic-aromatic interactions estimated from the binding of MTX and 6,7-dimethylpteridine are ca. $12.6 \mathrm{~kJ} \mathrm{~mol}^{-1}$. Since the salt bridge formed between N-1 and N-2 of MTX and Asp-27 may contribute ca. $7.5 \mathrm{~kJ} / \mathrm{mol}^{-1}$ to the drugs binding affinity (8), hydrophobic interactions are at least as important determinants in the tight binding of MTX.

The more complex question of the relationship between substrate/product binding and enzymic catalysis can also be scrutinized by mutagenesis. For at 
least five mutants including those at Phe-31, Leu-54, and Thr-113 there is a linear free energy relationship between the rate constant for hydride transfer and the dissociation constant for $\mathrm{H}_{2} \mathrm{~F}$ plotted logarithmically (20). In essence there is ca. a $8.4 \mathrm{~kJ} \mathrm{~mol}^{-1}$ increase in the free energy of activation for the chemical step for every $4.2 \mathrm{~kJ} \mathrm{~mol}^{-1}$ decrease in the free energy of $\mathrm{H}_{2} \mathrm{~F}$ dissociation. In short the weaker ground state binding of the substrate translates into a slower rate of hydride transfer, an example of a loss in ground state as well as a greater loss in transition state stabilization (21). The weaker binding of $\mathrm{H}_{2} \mathrm{~F}$ is also paralleled in weaker $\mathrm{H}_{4} \mathrm{~F}$ binding so that the dissociation rate constant for this product no longer may be rate limiting.

Particularly informative is the Leu-54 to Gly-54 mutation, ca. $18 \AA$ away from the locus of the hydride transfer, that decreases the rate of the chemical step from $950 \mathrm{~s}^{-1}$ to $14 \mathrm{~s}^{-1}$ and increases $K_{\mathrm{D}}\left(\mathrm{H}_{2} \mathrm{~F}\right)$ from $0.21 \mu \mathrm{mol} / 1$ to $140 \mu \mathrm{mol} / 1$ (ca. $16.7 \mathrm{~kJ} / \mathrm{mol}^{-1}$ ) (22). If the Gly substituent is viewed as too extreme a deletion, replacement of Leu-54 with Ile surprisingly produces an analogous change in the rate of the hydride transfer step $\left(30 \mathrm{~s}^{-1}\right)$ (23). The exquisite sensitivity of the locus to a single methyl group in the active site cannot at present be definitively rationalized; however an attractive hypothesis views bound $\mathrm{H}_{2} \mathrm{~F}$ as distributing between two conformers, one of which may be an unproductive complex akin to bound MTX whose equilibrium position is tuned by various residues. Moreover $a b$ initio calculations suggest the pathway for hydride transfer to be exquisitely sensitive to the distance and angle between the participating atoms, i. e. changes on the order of less than $1 \AA$ can decrease rates by factors of $10^{2}-10^{3}(24)$.

\section{Comparison of Mutiple Active Site Changes (E. coli and $L$. casei DHFR)}

The detailed kinetic description of the E. coli DHFR catalyzed reaction provided an opportunity to evaluate the efficiency of this process. Given the definition of the kinetic sequence under physiological conditions (at concentrations for NADPH/NADP ${ }^{+}$and $\mathrm{H}_{2} \mathrm{~F}$ / $\mathrm{H}_{4} \mathrm{~F}$ approximating those of the $E$. coli cell) the turnover at steady state and its reciprocal the reaction flux were calculated (25). A maximum hypothetical catalytic turnover also can be computed after imposing $\mathrm{H}_{2} \mathrm{~F}$ diffusion to the $\mathrm{E} \cdot \mathrm{NADPH}$ complex (the predominant form of the enzyme in the cell) as the only rate-limiting step. At physiological conditions the $E$. coli DHFR would be operating at ca. $15 \%$ efficiency, suggesting that the free energy cost of the various steps has been well optimized (20). Yet com- puter simulation revealed that there were a large number of pathways that efficiently solve the energetics of the reduction within the thermodynamic constraints imposed by the overall reaction equilibrium and the diffusional barrier of the enzyme/substrate combination. For example, the internal equilibrium constant $\left(\mathrm{E} \cdot \mathrm{NADPH} \cdot \mathrm{H}_{2} \mathrm{~F}: \mathrm{E} \cdot \mathrm{NADP}^{+} \cdot \mathrm{H}_{4} \mathrm{~F}\right)$ can be varied by $10^{3}$ with less than a two-fold change in turnover maintaining the uniform binding constraint (26). Two related questions are prompted by the above calculations: i) is the catalytic process exhibited by the $E$. coli DHFR also employed by DHFRs from other species; ii) how many and what kinds of active site changes can be tolerated while still maintaining high catalytic efficiency?

The x-ray crystallographic structure of the active site amino acids of the $E$. coli and $L$. casei DHFRs were overlaid using MTX as a center for alignment resulting in a base ensemble of 40 amino acids within $5 \AA$ of the drug. The active site equivalence was computed to be $55 \%$; those amino acids that were either identical or have backbone interactions only were deemed equivalent. Despite these differences comparison of the total solvent accessible surfaces of the two proteins within this $5 \AA$ radius showed them to be within $7 \%$ of each other and the protein surfaces to be remarkably congruent. However, several prominent changes (E. coli: L. casei) Ala-29: His-20; Leu-36: Val-35; Ile50: Phe-49; and Arg-52: Lys-51 result in discernible differences in the positioning of the glutamate and benzoyl residues. A similar overlay was constructed for the NADPH binding site after positioning the NADPH in the E. coli site by analogy to its known position in the $L$. casei. In this case an active site ensemble of 58 amino acids $7 \AA$ from the cofactor were counted with a $72 \%$ equivalence based on the above criteria. The close similarity in this section of the active site surface between the two species is shown in figure 5 despite the amino acid differences and the fact that the NADPH was modeled into the $E$. coli structure without changes in side chain positions.

Elucidation of the kinetic sequence for the $L$. case DHFR using the stopped-flow methods described earlier revealed the same features as reported for the $E$. coli $(20,27)$. Most importantly when the individual rate constants are expressed in terms of free energy the differences for each step (E. coli: $L$. casei) are within $4 \mathrm{~kJ} \mathrm{~mol}^{-1}$ equivalent to that of the $E$. coli enzyme. These free energy differences for the two enzymes are depicted for the bound species in figure 6 .

It is apparent that an active site with multiple amino acid changes can result in similar kinetic sequences and catalytic efficiencies. Since single mutations can 

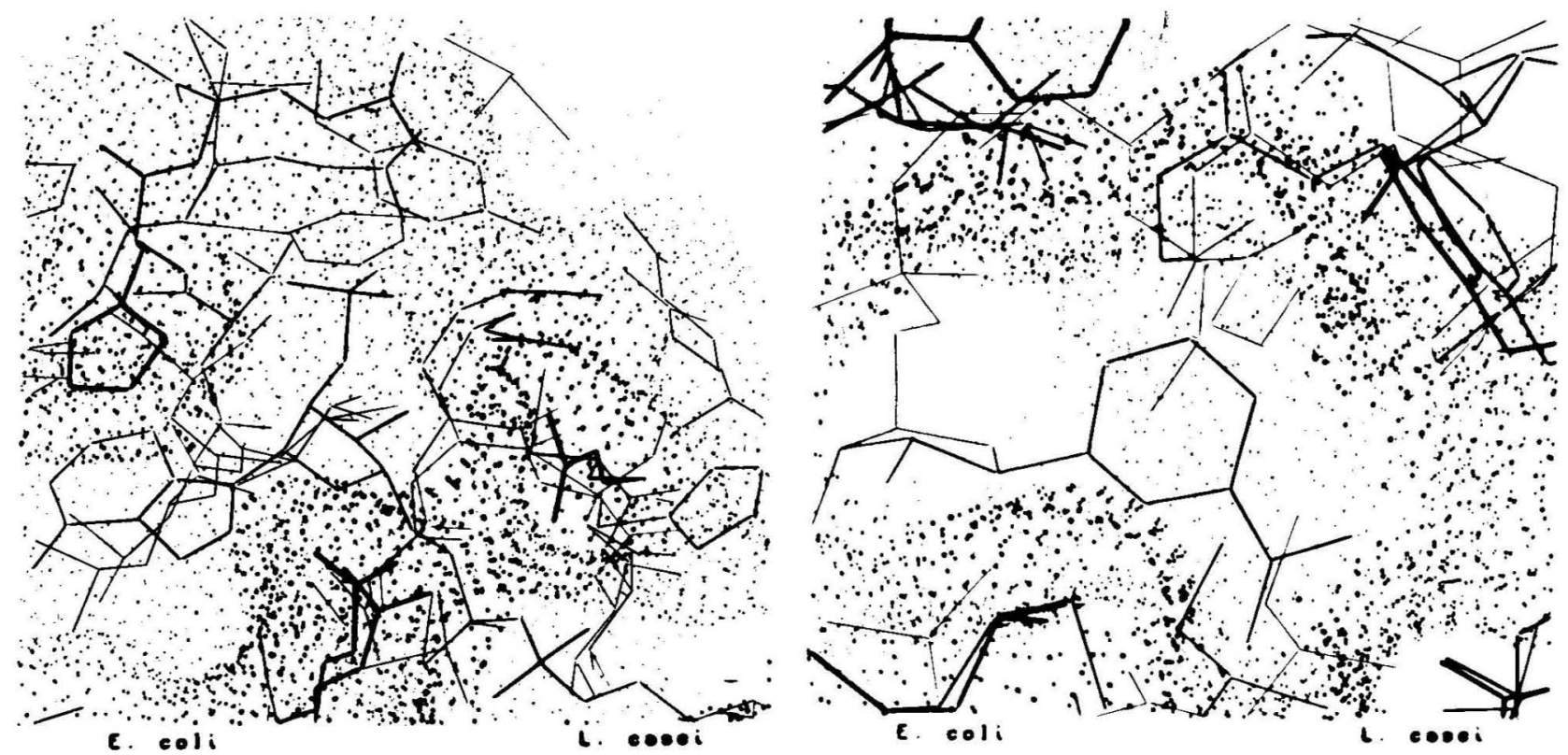

Figure 5. Superpositioning of the active sites of the E. coli and L. casei DHFRs with NADPH as a center for alignment. All residues within $7 \AA$ were included. Some key residues that are visible in the left hand panel (adenine portion) are $(E$. coli: $L$. casei); Gln-65: Gln-65 (top); Arg-44:Arg-43 (middle right); Arg-98: Gln-101 (bottom); and Ser-64: His-64 (middle left). In the right hand panel (nicotinamide portion) the key residues are (E. coli: L. casei); Ile-94: Ala-97 (upper right); Phe-31: Phe-30 (middle right); Asp-27: Asp-26 (lower right); and Ile-50: Phe-29 (upper left). These residues are depicted using BIOGRAF*.

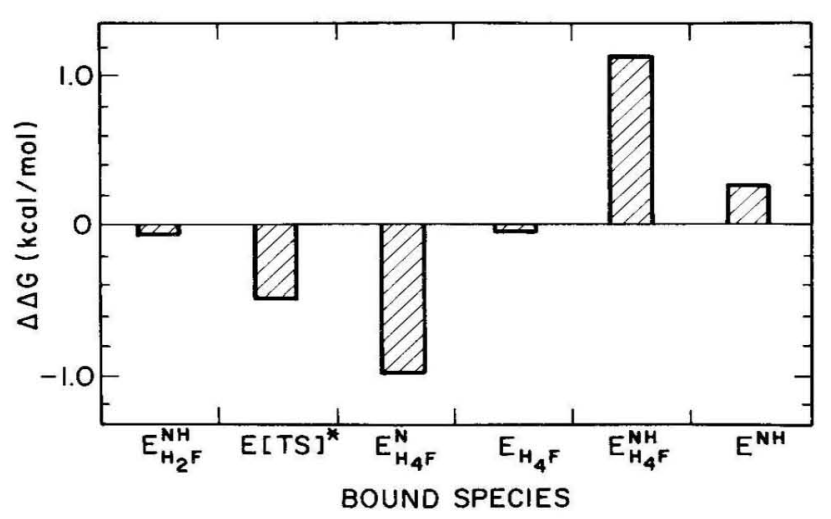

Figure 6. Plot of $\Delta \Delta G$ (E. coli minus L. casei) calculated from equilibrium constants vs. various bound species that are identified in figure 2 . $E[\mathrm{TS}]^{*}$ represents the transition state for the chemical hydride transfer step calculated from the forward rate constant. All free energy values are $\mathrm{pH}$ independent and are measured using standard state concentrations of $1 \mathrm{~mol} / 1$ $\mathrm{NADPH}$ and $\mathrm{H}_{2} \mathrm{~F} ; t=25^{\circ} \mathrm{C} ; 1 \mathrm{kcal}=4.1868 \mathrm{~kJ}$.

result in marked loss in catalytic activity, i. e. Leu-54 to Ile-54, it is reasonable to presume that some of these changes must be compensatory; others are probably neutral. One may hypothesize that the active site residues act as an ensemble to create a unique surface with the observed novel kinetic properties. The answer to the rhetorical question why this surface and not others may lie not only in the need for catalytic efficiency within the cell milieu but also in the stereochemical requirements of binding these substrates and in curtailing inhibition by others and the demands for stably folding a given primary sequence into this cavity shape. One may also speculate that residues strictly conserved during evolution may not be changed because no compensatory mutation exists that satisfies all these three requirements within the cell.

\section{Acknowledgement}

We thank W. A. Goddard III for use of the computer graphics facility for graphical display.

\section{References}

1. Blakley, R. L. (1985) in Folates and Pterins (R. L. Blakley and S. J. Benkovic, eds.) Wiley, New York.

2. Matthews, D. A., Alden, R. A., Bolin, J. T., Filman, D. J., Freer, S. T., Hamlin, R., Hol, W. G. J., Kisliuk, R. L., Pastore, E. J., Plante, L. T., Xuong, N.-H. \& Kraut, J. (1978) J. Biol. Chem. 253, 6946-6954.

3. Matthews, D. A., Alden, R. A., Freer, S. T., Xuong, N.H. \& Kraut, J. (1979) J. Biol. Chem. 254, 3144-4154.

4. Bolin, J. T., Filman, D. J., Matthews, D. A., Hamlin, R. C. \& Kraut, J. (1982) J. Biol. Chem. 257, 13650-13662.

5. Filman, D. J., Bolin, J. T., Matthews, D. A. \& Kraut, J. (1982) J. Biol. Chem. 257, 13663-13672.

6. Charlton, P. A., Young, D. W., Birdsall, B., Feeney, J. \& Roberts, G. C. K. (1979) J. Chem. Soc. Chem. Commun. $992-924$. 
7. Fierke, C. A., Kuchta, R. D., Johnson, K. A. \& Benkovic, S. J. (1987) Cold Spring Harbor Symp. on Quant. Biol. 52 , $631-638$.

8. Howell, E. E., Villafranca, J. E., Warren, M. S., Oatley, S. J. \& Kraut, J. (1986) Science 231, 1123-1128.

9. Chen, J.-T., Taira, K., Tu, D. D. \& Benkovic, S. J. (1987) Biochemistry 26, 4093-4100.

10. Taira, K., Chen, J.-T., Fierke, C. A. \& Benkovic, S. J. (1987) Bull. Chem. Soc. Jpn. 60, 3025-3030.

11. Gready, J. E. (1985) Biochemistry 24, $4761-4766$.

12. Howell, E. E., Warren, M. S., Booth, C. L. J., Villafranca, J. E. \& Kraut, J. (1987) Biochemistry 26, 8591-8598.

13. Perry, K. M., Onuffer, J. J., Touchette, N. A., Herndon, C. S., Gittleman, M. S., Matthews, C. R., Chen, J.-T., Mayer, R. J., Taira, K., Benkovic, S. U., Howell, E. E. \& Kraut, J. (1987) Biochemistry 26, 2674-2682.

14. Adams, J. A. (1987). Unpublished results.

15. Russel, A. J. \& Fersht, A. R. (1987) Nature 328, 496-500.

16. Fersht, A. R. (1987) Trends Biochem. Sci. 12, 301-304.
17. Williams, J. W., Morrison, J. F. \& Duggleby, R. G. (1979) Biochemistry 18, 2567-2573.

18. Blakley, R. L. \& L. Cocco (1985) Biochemistry 24, 47724777.

19. Taira, K. \& Benkovic, S. J. (1987) J. Med. Chem. 31, $129-137$.

20. Benkovic, S. J., Fierke, C. A. \& Naylor, A. M. (1987) Science 239, 1105-1110.

21. Jencks, W. P. (1975) Advances in Enzymology 43, 219410.

22. Mayer, R. J., Chen, J.-T., Taira, K., Fierke, C. A. \& Benkovic, S. J. (1986) Proc. Natl. Acad. Sci. 83, 7718-7720 .

23. Murphy, D. J. (1987) Unpublished results.

24. Wu, Y. D. \& Houk, K. N. (1987) Personal communication.

25. Fierke, C. A., Johnson, K. A. \& Benkovic, S. J. (1987) Biochemistry 26, $4085-4092$.

26. Albery, W. J. \& Knowles, J. R. (1976) Biochemistry 15, $5631-5640$.

27. Andrews, J. (1988) Unpublished results. 